$\mathrm{OH}$ and the University of Iowa, Iowa City, IA. She had received Depakote from 10 years 7 months of age in doses ranging from $750 \mathrm{mg} / \mathrm{day}$ (17 $\mathrm{mg} / \mathrm{kg} / \mathrm{day}$ ) to $1,500 \mathrm{mg} / \mathrm{day}(36 \mathrm{mg} / \mathrm{kg} /$ day) and a maximum $\mathrm{VPA}$ level of $135.5 \mathrm{ug} / \mathrm{dl}$. Her growth rate during treatment decreased to $1.9 \mathrm{~cm} / \mathrm{yr}$ compared to $4.9 \mathrm{~cm} / \mathrm{yr}$ prior to treatment. When treatment was discontinued, normal pubertal growth and maturation resumed with a growth rate of 10 $\mathrm{cm} / \mathrm{yr}$. Cranial MRI with gadolinium and CT were normal (Cook JS et al. Pubertal arrest associated wih valproic acid therapy. Pediatr Neurol May/June 1992; 8 :229-231). (Correspondence: Dr. Cook, Department of Pediatrics, Wright State University, Dayton, $\mathrm{OH} 45402$.)

COMMENT. The authors recommend that children and adolescents on VPA therapy should have height, weight and Tanner staging of genitalia and pubic hair assessed at each clinic visit to detect arrest of growth and development.

A causal association between maternal use of VPA and fetal spina bifida has been reported (Omtzigt JGC et al. The risk of spina bifida aperta after first trimester exposure to valproate in a prenatal cohort. Neurology 1992; 42 (suppl 5):119-125.) Of 261 pregnant women receiving antiepileptic drugs, $6.3 \%$ of fetuses with VPA exposure had spina bifida aperta, whereas other antiepileptic drugs were not implicated in this developmental abnormality. High serum levels of VPA in the mother may have increased the risk which might be diminished by reduction of the daily dose.

\title{
OCCIPITAL LOBE SEIZURES AND CELIAC DISEASE
}

The electroclinical ictal findings of 4 epileptic patients with clinically asymptomatic celiac disease are reported from the University of Bologna, Italy. All patients had paroxysmal visual manifestations and EEG seizure discharges arising from the occipital lobe. CT in 2 patients showed occipital calcifications. A gluten-free diet was not beneficial and polytherapy with antiepileptic drugs aggravated the seizures in 3 cases. Epilepsy and evidence of steatorrhea and growth failure were present in early childhood. The diagnosis of celiac disease was made by tests for serum folate level, a dzylose absorption test, anti-gliadin antibody test and jejunal biopsy which showed villous atrophy (Ambrosetto $\mathrm{G}$ et al. Occipital lobe seizures related to clinically asymptomatic celiac disease in adulthood. Epilepsia May/June $1992 ; 33: 476-481$ ). (Reprints: Professor G. Ambrosetto, Institute of Neurology, Via Ugo Foscolo 7, 40123, Bologna, Italy.)

COMMENT. The same authors had investigated 10 patients with epilepsy and occipital cerebral calcification and had found evidence of celiac disease in 6. All patients with epilepsy and posterior cerebral calcification should be investigated for possible celiac disease. (Gobbi G, Ambrosetto $\mathrm{P}$ et al. Celiac disease, posterior cerebral calcifications and epilepsy. Brain Dev Jan 1992; 14:23-29.) 